\title{
Temporal Stability of Dominant Frequency as Predictor of Atrial Fibrillation Recurrence
}

\author{
Alejandro Costoya-Sánchez ${ }^{1}$, Andreu M Climent ${ }^{1,2}$, Ismale Hernández-Romero ${ }^{3}$, Alejandro Liberos ${ }^{1}$, \\ Francisco Fernández-Avilés ${ }^{2}$, Sanjiv M Narayan ${ }^{4}$, Felipe Atienza ${ }^{2}$, María S Guillem ${ }^{1}$, Miguel \\ Rodrigo ${ }^{1}$ \\ ${ }^{1}$ ITACA Institute, Universitat Politècnica de València, Valencia, Spain \\ ${ }^{2}$ Hospital General Universitario Gregorio Marañón, Madrid, Spain; ${ }^{3}$ Department of Signal Theory \\ and Communications, Rey Juan Carlos University, Spain; ${ }^{4}$ Cardiovascular Department, Stanford \\ University, CA, USA;
}

\begin{abstract}
Catheter ablation is one of the main therapies for restoring sinus rhythm in patients with atrial fibrillation $(A F)$, yet AF termination ratios are far from satisfactory. The goal of this work is to study if temporal stability of dominant frequencies (DFs) of electrograms (EGMs) can be used as predictor of $A F$ recurrence.

EGMs were recorded from 29 AF patients using 64pole basket catheters during the ablation procedure. DFs before ablation were obtained for 4-second overlapping fragments of EGM recordings with a $0.4 \mathrm{~s}$ shift, and their temporal stability was evaluated for short-term (between 8 and $12 \mathrm{~s}$ ) and long-term time intervals $(>5 \mathrm{~min})$. Patients were classified as $A F(N=15)$ if sinus rhythm was not maintained in a 12-month post-ablation followup, and AF free otherwise $(N=14)$.

Significant differences were found in the short-term analysis between $A F$ free and $A F$ patients for the difference between the mode value in DFs ( $p=0.045)$, as well as for the long-term analysis for the normalized average between DFs $(p=0.028)$ and the average between DFs ( $p=0.043)$. More stable values were found for $A F$ free patients for all statistically significant metrics.

Short- and long-term temporal stability of DF values of EGM signals were found to be associated with the 12month success rate of ablative therapies of AF patients.
\end{abstract}

\section{Introduction}

Catheter-based ablative therapy is one of the main approaches currently applied to restore sinus rhythm in patients suffering atrial fibrillation (AF), and specifically pulmonary veins isolation has become the recommended procedure. However, long-term success rates of ablation procedures are far from satisfactory and varies between patients [1]. Therefore, characterization of AF using clinical parameters has become increasingly important as a predictor of the outcome of ablative therapies.

Previous works have focused on the analysis of signal parameters obtained from intracardiac electrograms (EGM) as predictors of termination of AF using spectral information [2,3]. Nevertheless, temporal variability of EGM signal parameters has yet to be investigated and evaluated as a potential predictor of ablation outcomes.

This goal of this work is to study whether temporal stability of dominant frequency (DF) values obtained from intracardiac EGMs is associated with $\mathrm{AF}$ termination ratios and could be applied to patient selections strategies.

\section{Methodology}

29 patients from Hospital GU Gregorio Marañón were referred for AF ablation were included in this study. Intracardiac electrograms and atrial anatomy. Intracardiac electrograms and atrial anatomy were obtained using 64electrode intracavitary basket catheters (Constellation, Boston Scientific, Natick, MA, USA) introduced via the right femoral vein and guided by an electroanatomical navigation system (Ensite NavX System version 8.0, St. Jude Medical, Minneapolis, Minnesota). This study was approved by the ethics committee of the hospital, and all patients gave informed consent.

Patients were classified as $\mathrm{AF}(\mathrm{N}=15)$ if sinus rhythm was not maintained in a 12-month post-ablation followup, and $\mathrm{AF}$ free otherwise $(\mathrm{N}=14)$. 


\section{1. $\quad$ EGM preprocessing}

EGM signals underwent average QRS substraction and were filtered using a 2 to $20 \mathrm{~Hz}$ band-pass filter followed by Botteron filtering [4]. Power Spectral Density (PSD) was obtained for 4-second overlapping fragments of EGM recordings with a $0.4 \mathrm{~s}$ shift. PSD was calculated with the Welch periodogram using a Hamming window with $50 \%$ overlap.

\subsection{DF stability metrics}

DF stability metrics were calculated using the DF values from pairs of EGM fragments obtained from the same EGM signal. The metrics were: rate of EGM signals with a bigger difference in DF value than $0.5 \mathrm{~Hz}$, the average difference between DFs, the normalized average difference between DFs, the average difference between HDF value (obtained as the 95 percentile of the DF values [5]), the difference between the median value of DFs, the difference between the mode value of DFs and the difference between the average value of the DFs.

\subsection{Stability evaluation criteria}

Stability analysis was performed according to two different criteria. For the short-term analysis, DF stability metrics from EGM fragments separated between 8 and 12 $\mathrm{s}$ were calculated. Mean value of the stability metrics was calculated for each patient. Due to lack of $12 \mathrm{~s}$ recordings from some episodes, only 23 patients were considered for the analysis (11 AF free patients, $12 \mathrm{AF}$ patients).

\section{A DF map for AF free patient}

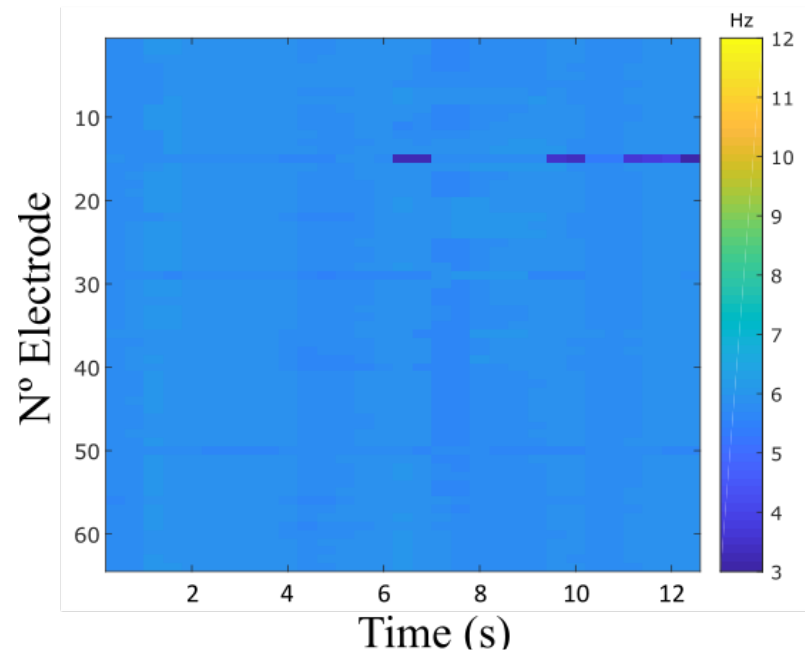

For the long-term analysis, DF stability metrics were calculated from EGM recorded from different episodes separated more than 5 min for each patient. Only patients with 2 or more recorded episodes were considered for this analysis (10 AF free patients, $9 \mathrm{AF}$ patients).

Statistical analysis between groups of patients was performed for each stability metric. Normality was tested using a Kolmogorov-Smirnov test (alpha $=0.05$ ), and comparison of data between groups was performed using Student's t-test when normality was assumed, and Wilcoxon test otherwise.

\section{Results}

\subsection{Short-term analysis}

DF stability metrics presented significant differences between $\mathrm{AF}$ and $\mathrm{AF}$ free patients in the short-term analysis. In Figure 1, DF value maps and their temporal evolution are showed for an example of an $\mathrm{AF}$ free and an AF patient. It can be observed that temporal stability of $\mathrm{DF}$ values is higher for the AF free patient.

Short-term analysis presented significant differences for some of the stability metrics compared, which can be appreciated in Table 1 and Figure 2 (top). Difference between the mode value of the DFs was lower in patients that reamined $\mathrm{AF}$ free 12 months after ablation (AF free $0.42 \pm 0.43 \mathrm{~Hz}$ vs. AF $0.95 \pm 0.68 \mathrm{~Hz}, \mathrm{p}=0.045$ ). Other stability metrics that also presented statistical differences between groups were the diffence between the average value of the DFs (AF free $0.34 \pm 0.21 \mathrm{~Hz}$ vs. AF $0.60 \pm 0.40$ $\mathrm{Hz}, \mathrm{p}=0.053)$ and the normalized average difference between DFs (AF free $0.15 \pm 0.09 \mathrm{~Hz}$ vs. AF $0.23 \pm 0.12$ $\mathrm{Hz}, \mathrm{p}=0.069)$.

\section{B DF map for AF patient}

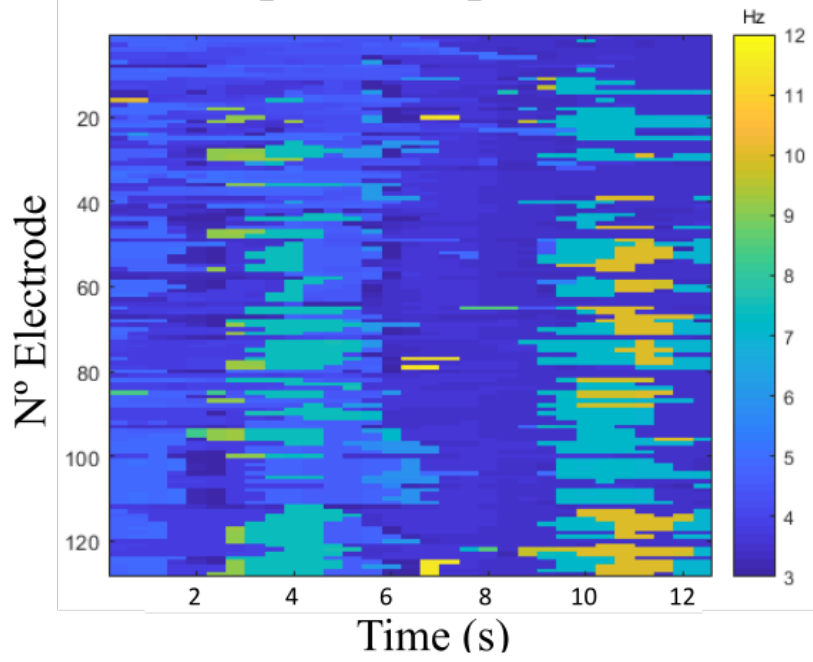

Figure 1. Temporal evolution of dominant frequency before ablation of each electrode. A. DF map for AF free patients. B. DF map for AF patients. 


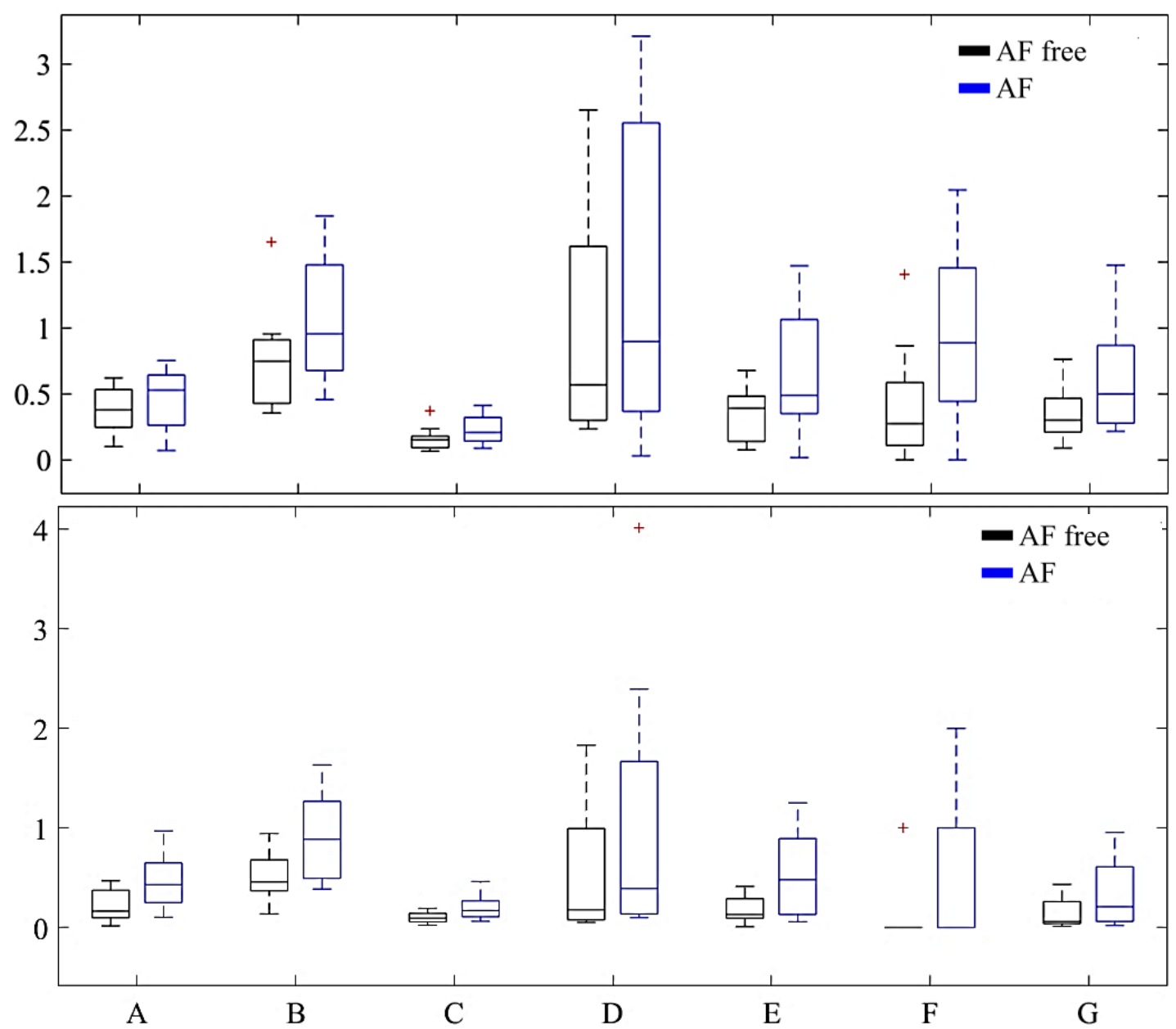

Figure 2. Box-whiskers graphs of DF stability metrics obtained from intracardiac EGMs for short-term analysis (top) and long-term analysis (bottom). A. Rate of EGM signals with a bigger difference ni DF value than $0.5 \mathrm{~Hz}$. B. Average difference between DFs. C. Normalized average difference between DFs. D. Average difference between HDF value. E. Difference between the median value of DFs. F. Difference between the mode value of DFs. G. Difference between the average value of DFs.

Table 1. p-values for short-term stability metrics.

\begin{tabular}{l|l} 
Stability metric & $\mathbf{p}$-value \\
\hline $\begin{array}{l}\text { Rate of EGM signals with a bigger } \\
\text { difference ni DF value than } 0.5 \mathrm{~Hz}\end{array}$ & 0.280 \\
\hline Average difference between DFs & 0.103 \\
\hline $\begin{array}{l}\text { Normalized average difference } \\
\text { between DFs }\end{array}$ & 0.069 \\
\hline $\begin{array}{l}\text { Average difference between HDF } \\
\text { value }\end{array}$ & 0.644 \\
$\begin{array}{l}\text { Difference between the median } \\
\text { value of DFs }\end{array}$ & 0.132 \\
$\begin{array}{l}\text { Difference between the mode value } \\
\text { of DFs }\end{array}$ & 0.045 \\
\hline $\begin{array}{l}\text { Difference between the average } \\
\text { value of DFs }\end{array}$ & 0.053
\end{tabular}

\subsection{Long-term analysis}

Significant differences were found as well in the longterm analysis between $\mathrm{AF}$ and $\mathrm{AF}$ free patients for some of the stability metrics compared, which can be appreciated in Table 2 and Figure 2 (bottom). Lower values were obtained in some of these metrics for AF free patients, such as in normalized average difference between DFs (AF free $0.10 \pm 0.05 \mathrm{~Hz}$ vs. AF $0.19 \pm 0.10$ $\mathrm{Hz}, \mathrm{p}=0.028$ ) and average difference between DFs (AF free $0.53 \pm 0.22 \mathrm{~Hz}$ vs. AF $0.81 \pm 0.40 \mathrm{~Hz}, \mathrm{p}=0.043$ ). Other evaluated metrics that showed statistically significant difference were the differece between the mode value of DFs $(p=0.069)$, the difference between the median value of DFs $(p=0.075)$ and the rate of EGM signals with a bigger difference in DF value than $0.5 \mathrm{~Hz}(\mathrm{p}=0.075)$. 
Table 2. p-values for long-term stability metrics.

\begin{tabular}{l|l} 
Stability metric & p-value \\
\hline $\begin{array}{l}\text { Rate of EGM signals with a bigger } \\
\text { difference in DF value than 0.5 Hz }\end{array}$ & 0.075 \\
\hline $\begin{array}{l}\text { Average difference between DFs } \\
\text { Normalized average difference } \\
\text { between DFs }\end{array}$ & 0.043 \\
\hline $\begin{array}{l}\text { Average difference between HDF } \\
\text { value }\end{array}$ & 0.278 \\
\hline $\begin{array}{l}\text { Difference between the median } \\
\text { value of DFs }\end{array}$ & 0.075 \\
\hline $\begin{array}{l}\text { Difference between the mode value } \\
\text { of DFs }\end{array}$ & 0.069 \\
\hline $\begin{array}{l}\text { Difference between the average } \\
\text { value of DFs }\end{array}$ & 0.113
\end{tabular}

\section{Dicussion}

This work presents a novel study in which the DF temporal dynamics were evaluated in relation with the success of ablative therapies for AF patients. DF temporal stability metrics were obtained from intracardiac EGM recordings from $29 \mathrm{AF}$ patients have proven to be associated with the success of ablative therapies for AF patients 12 months after ablation.

Short-term analysis proved that DF stability information capable of characterizing AF persistance after ablation can be acquired from EGM recordings as short as $12 \mathrm{~s}$. Moreover, patients in which ablation was successful (AF free patients) showed more stable values for some of the stability metrics evaluated. Statistically significant differences were found for the difference between the mode value of DFs $(p=0.045)$, the diffence between the average value of the $\mathrm{DFs}(\mathrm{p}=0.053)$ and the normalized average difference between DFs $(\mathrm{p}=0.069)$.

Results obtained with long-term analysis proved that association between ablation success rate and DF stability can be found for a higher number of evaluated metrics. This indicates that differences in DF stability between groups become more pronounced for long time intervals ( $>5 \mathrm{~min}$ ). Long-term showed that DF values are more temporally stable for $\mathrm{AF}$ free patients for some of these metrics, such as normalized average between DFs $(\mathrm{p}=0.028)$ and average difference between DFs $(\mathrm{p}=0.043)$. Other metrics that showed statistically significant difference between patient groups were the mode value of DFs $(p=0.069)$, the difference between the median value of DFs $(\mathrm{p}=0.075)$ and the rate of EGM signals with a bigger difference in $\mathrm{DF}$ value than $0.5 \mathrm{~Hz}(\mathrm{p}=0.075)$.

This study, in line with others previously published $[2,3]$, has shown that the spectral domain of the EGM signal obtained prior to AF ablation contains information of the recurrence of such therapies. Moreover, analysis of the temporal evolution of such spectral information, and specifically, DF values, has been found to be associated with the recurrence of the arrythmia. Therefore, inclusion of DF-based stability parameters allows the improvement of the patient selection strategy for ablative therapies.

\section{Conclussion}

Temporal stability metrics of DF values obtained from intracardiac EGM recordings have proven to be associated with the success of ablative therapies for AF patients. Comparison of DF stability metrics in short- and long-term showed that AF patients that mantained sinus rhythm 12 months after ablation presented more stable $\mathrm{DF}$ values than patients recurring in $\mathrm{AF}$.

\section{Acknowledgments}

This work was supported by the Instituto de Salud Carlos III FEDER (DTS16/00160; PI16/01123; PI17/01059; PI17/01106), the EIT-HEALTH 19600 AFFINE and the Valencian Regional Government (AICO2018).

\section{References}

[1] Chao T et al., "Clinical outcome of catheter ablation in patients with nonparoxysmal atrial fibrillation," Circ Arrhythm Electrophysiol, vol. 5, no. 3, pp. 514-520, May 2012.

[2] Szilágyi J et al., "Surface ECG and intracardiac spectral measures predict atrial fibrillation recurrence after catheter ablation," J Cardiovasc Electrophysiol, vol. 20, no. 10, pp. 1371-1378, Oct. 2018.

[3] Garibaldi $\mathrm{M}$ et al. "Predicting catheter ablation outcome in persisten atrial fibrillation using atrial dominant frequency and related spectral features," Conf Proc IEEE Eng Med Biol Soc, pp. 613-616

[4] Castells F et al., "On the preprocessing of atrial electrograms in atrial fibrillation: understanding Botteron's approach," Pacing Clin Electrophysiol, vol. 37, no. 2, pp. 133-143, Feb. 2014.

[5] Rodrigo $\mathrm{M}$ et al., "Highest dominant frequency and rotor positions are robust markers of driver location during noninvasive mapping of atrial fibrillation: A computational study," Heart Rhythm, vol. 14, no. 8, pp. 1224-1233, Aug. 2017.

Address for correspondence:

Miguel Rodrigo.

ITACA Institute, Universitat Politècnica de València. mirodbor@teleco.upv.es 